\title{
Allelic diversity of metallothionein in Orchesella cincta (L.): traces of natural selection by environmental pollution
}

\author{
MJTN Timmermans, J Ellers and NM Van Straalen \\ Department of Animal Ecology, Vrije Universiteit Amsterdam, Amsterdam, The Netherlands
}

\begin{abstract}
The advances made in statistical methods to detect selection from DNA sequence variation has resulted in an enormous increase in the number of studies reporting positive selection. However, a disadvantage of such statistical tests is that often no insight into the actual source of selection is obtained. Finer understanding of evolution can be obtained when those statistical tests are combined with field observations on allele frequencies. We assessed whether the metallothionein $(m t)$ gene of Orchesella cincta (Collembola), which codes for a metal-binding protein, is subject to selection, by investigating alleles and allele frequencies among European metalstressed and reference populations. Eight highly divergent alleles were resolved in Northwest Europe. At the nucleotide level, a total of 51 polymorphic sites (five of them implying amino-acid changes) were observed. Although statistical tests applied to the sequences alone showed no indication of
\end{abstract}

selection, a G-test rejected the null hypothesis that alleles are homogeneously distributed over metal-stressed and reference populations. Analysis of molecular variance assigned a small, but significant amount of the total variance to differences between metal-stressed and non-stressed populations. In addition, it was shown that metal-stressed populations tend to be more genetically diversified at this locus than non-stressed ones. These results suggest that the $m t$ gene and its surrounding DNA region are affected by environmental metal contamination. This study illustrates that, in addition to statistical tests, field observations on allele frequencies are needed to gain understanding of selection and adaptive evolution.

Heredity (2007) 98, 311-319. doi:10.1038/sj.hdy.6800942; published online 21 February 2007

Keywords: metal tolerance; metallothionein; selection; allelic diversity; Orchesella cincta

\section{Introduction}

Advances in molecular techniques have made it possible to screen for functional polymorphisms in coding regions of the genome and link them to fitness (Vasemagi and Primmer, 2005). This approach allows us to study the genetic mechanisms through which natural selection promotes adaptation. Studies describing changes in allele frequencies induced by natural selection are numerous, ranging from bacteria (e.g. antibiotic resistance) to mammals. A well-known example comes from insecticide (organophosphate) resistance in the mosquito Culex pipiens caused by a modified acetylcholinesterase (Raymond et al., 2001). Lenormand et al. (1999) showed that the frequency of 'resistance' alleles was higher in organophosphate-treated areas, and tended to decrease with distance from those treated sites. A mammalian example of environmentally induced shifts in allele frequencies is given by Nachman et al. (2003). These authors observed that the frequency of allelic variants of the melanocortin-1-receptor gene of pocket mice that

Correspondence: Dr MJTN Timmermans, Department of Animal Ecology, Vrije Universiteit Amsterdam, De Boelelaan 1085, Amsterdam 1081HV, The Netherlands.

E-mail: Martijn.Timmermans@falw.vu.nl

Received 12 January 2006; revised 18 July 2006; accepted 4 December 2006; published online 21 February 2007 result in dark or light coat color are related to the dark or light color of the substrate on which they live. This correlation between mutations in the melanocortin-1receptor (coat color) and substrate color is thought to originate from natural selection induced by owl predation.

In addition to changes in coding regions of genes, phenotypic differences may result from gene duplications and from quantitative differences in gene expression (changes in promoter regions and trans-acting factors) (Wray et al., 2003). For example, laboratory experiments assessing increased metal tolerance in Drosophila melanogaster, revealed that a duplication of a metallothionein $(m t)$ gene $(M t n A)$ resulted in increased $M t n A$ mRNA levels and increased tolerance to copper and cadmium (Maroni et al., 1987). Other examples are given by the house fly (Musca domestica) and by $D$. melanogaster. For both species, overtranscription of a single gene is sufficient for insecticide resistance. Overexpression of CYP6D1 in $M$. domestica results in pyrethroid resistance (Liu and Scott, 1998); Cyp6g1 overexpression in D. melanogaster results in DDT resistance (Daborn et al., 2002).

Even if information on fitness of alternate genotypes for a locus is lacking, adaptive mutations can be unraveled. Over the last several years, new methods have been developed to detect selection from patterns of DNA sequence variation, comparing polymorphic sites, 
heterozygosity and genetic divergence with expectations of the neutral theory of molecular evolution (Nei and Kumar, 2000). The enhanced ability of statistical methods to detect traces of selection, in combination with the ease with which DNA sequences can now be obtained, has caused the number of studies reporting positive selection to grow remarkably in recent years (Ford, 2002). Analysis of these footprints of selection in the genome has made it clear that a surprisingly large percentage of substitutions are driven by adaptive evolution.

However, a disadvantage of studies on DNA sequence variation only is that often no insight into the actual source of selection is obtained. A review of 119 studies reporting positive selection based on statistical tests on DNA sequence variation, showed that the source of selection, as well as the acquired fitness benefits remained unknown (Ford, 2002). Therefore, these type of studies help us to obtain estimates of the frequency of selection, but do not always add to the understanding of the selective mechanisms that caused the genetic change. In contrast, when the function of a studied gene is known statistical tests on DNA sequences will enable the postulation of adaptive hypotheses, which can be tested by additional studies and observations (Ford, 2002). Such combined analyses should provide strong evidence for adaptive processes in the wild.

The study presented in this paper is an example of such a field study that attempts to link selective environments with molecular changes underlying adaptation. We test if a clearly measurable (directional) selection pressure leads to changes in the coding region of a gene of known function; the $m t$ gene of the springtail Orchesella cincta (Collembola). This gene provides a useful system for analyzing genetic effects of selection, empirically and statistically: in addition to the DNA sequence and the function of this gene, the selective force operating is known, namely environmental metal pollution.

Selection by environmental metal pollution is expected to be directional (Posthuma and van Straalen, 1993), often leading to populations exhibiting increased tolerance to metals (e.g. Timmermans et al., 2005b). The evolution of cadmium tolerance has been studied in close detail in natural populations of the springtail $O$. cincta. The genomic mechanism responsible for metal tolerance in O. cincta most likely involves increased $m t$ production. Upon dietary exposure to cadmium, O. cincta from a cadmium-polluted population (Plombières) showed significantly higher $m t$ gene upregulation compared to animals from an unpolluted reference population (Sterenborg and Roelofs, 2003). Roelofs et al. (2006) showed that this increased $m t$ expression is related to the presence of certain $m t$ promoter alleles. In their study three $m t$ promoter alleles (B, D1 and D2) had an increased occurrence in groups of animals showing high $m t$ expression. Furthermore, they demonstrated significant additive genetic variation in cadmium-induced $m t$ gene expression in a reference (non-metal stressed) O. cincta population (Roggebotzand, Flevoland, The Netherlands), which implies that this population would be able to respond to selection driven by cadmium.

Although the significance of the $m t$ gene in metal tolerance of $O$. cincta is not yet fully unraveled, it is obvious that the gene plays an important role in the stress-response after exposure to cadmium. If the $m t$ gene is indeed involved in metal tolerance, there may be selection for certain allelic variants of the gene at metalpolluted sites, for instance owing to differences in aminoacid composition (e.g. leading to a better metal-binding capacity). Alleles with a better binding capacity may replace other segregating alleles. Alternatively, changes in allele frequencies can be induced by genetic linkage to selected functional polymorphisms in the promoter region (Wray et al., 2003). We tested the hypothesis that the $m t$ gene is subject to selection in metal-stressed populations, resulting in changes in nucleotide composition and/or allele frequencies. To determine if selection by environmental metal pollution is acting at the $m t$ locus of O. cincta, 290 individuals from 15 European metal-stressed and reference populations (eight metalstressed and seven reference populations) were genotyped. We conducted several statistical tests on intraspecific sequence variation in the $m t$ gene and examined effects of natural selection by the distribution of alleles over populations.

\section{Materials and methods}

\section{Species description and sampling design}

$O$. cincta is a soil-dwelling arthropod species distributed across Europe. In total, 15 geographically distinct populations were sampled in Europe from August through September 2002 (Table 1) in a pairwise design: each environmentally stressed population was coupled to a reference population in the same geographical area (Figure 1). Metal-stressed and reference populations were chosen $10-95 \mathrm{~km}$ apart. To obtain more or less representative population estimates, animals for each population were collected at two sites within a distance of about $50 \mathrm{~m}$ apart (cf. Van der Wurff et al., 2003). Individuals were taken to the laboratory alive and kept 2 days without food to empty their gut and were then stored at $-80^{\circ} \mathrm{C}$. All metal-contaminated sites were chosen based on earlier ecotoxicological field studies (for additional information see Timmermans et al., 2005b).

\section{DNA extraction and polymerase chain reaction amplification}

Before DNA extraction, animals were rinsed in $80 \%$ ethanol. Total DNA was extracted using the Wizard SV Genomic DNA purification system (Promega, Madison, WI, USA). DNA was dissolved in $100 \mu \mathrm{l}$ nuclease-free water and stored at $-20^{\circ} \mathrm{C}$ until polymerase chain reaction (PCR) amplification. The sample size for each population is given in Table 1 . The $m t$ gene of $O$. cincta is a single copy gene (Sterenborg and Roelofs, 2003). Part of the gene was amplified using two primer pairs: MTF: 5'-ACTCTAAAAACTCAAgCAgCC-3' and 184R: 5'-TAC AACAAgTgggCgATTTgC- $3^{\prime}$ and 163F: $5^{\prime}$-ggCAAATCg CCCACTTgTT-3' and 265R: 5'-CCTTgCAgACACAATC TggACC-3'. Reaction volumes of $25 \mu \mathrm{l}$ contained $2.5 \mu \mathrm{l}$ $10 \times$ buffer, $0.6 \mathrm{U}$ Taq polymerase (MRC Holland, Amsterdam, The Netherlands), one $0.5 \mu \mathrm{M}$ of each primer, $0.5 \mathrm{mM}$ dNTP and $1 \mu \mathrm{l}$ template DNA. PCR was performed using $3 \mathrm{~min}$ at $95^{\circ} \mathrm{C}$ followed by 30 cycles of $15 \mathrm{~s}$ at $95^{\circ} \mathrm{C}, 45 \mathrm{~s}$ at $54^{\circ} \mathrm{C}$ and $30 \mathrm{~s}$ at $72^{\circ} \mathrm{C}$. A final elongation step of $10 \mathrm{~min}$ at $72^{\circ} \mathrm{C}$ was added. It has to be mentioned that later work showed that the MTF primer 
Table 1 Sample sizes $(\mathrm{N})$, allele frequencies, Ho, tests for HW equilibrium, genetic diversity and neutrality tests of partial $m t$ sequence of 15 European O. cincta populations

\begin{tabular}{|c|c|c|c|c|c|c|c|c|c|c|c|c|c|c|c|c|c|}
\hline \multirow[t]{2}{*}{ Population } & \multirow[t]{2}{*}{ Country } & \multirow[t]{2}{*}{$N$} & \multirow{2}{*}{$2 N$} & \multirow{2}{*}{$\begin{array}{l}\text { No. of } \\
\text { alleles }\end{array}$} & \multicolumn{8}{|c|}{ Allele frequencies } & \multirow[t]{2}{*}{ Ho } & \multirow{2}{*}{$\begin{array}{l}\text { HW } \\
\text { P-value }\end{array}$} & \multirow{2}{*}{$\begin{array}{c}\text { Haplotype } \\
\text { diversity } \\
\text { Gene diversity }\end{array}$} & \multirow{2}{*}{$\begin{array}{l}\text { Nucleotide } \\
\text { diversity } \\
\text { excl. gaps }\end{array}$} & \multirow[t]{2}{*}{ Tajima's D } \\
\hline & & & & & $A$ & $B$ & C & $D$ & E & $F$ & $G$ & $H$ & & & & & \\
\hline Brasschaat & Belgium & 20 & 40 & 5 & 60.0 & & 20.0 & 7.5 & & 2.5 & & & 0.60 & 0.84 & 0.599 & 0.0197 & $0.9419 \mathrm{NS}$ \\
\hline Hoboken & Belgium & 16 & 32 & 4 & 62.5 & 6.3 & 21.9 & 3.1 & & 6.3 & & & 0.38 & 0.05 & 0.570 & 0.0184 & 0.1186 NS \\
\hline Hecq & France & 19 & 38 & 3 & 71.1 & & 15.8 & 13.2 & & & & & 0.16 & $<0.01$ & 0.465 & 0.0192 & $0.7973 \mathrm{NS}$ \\
\hline Noyelles & France & 20 & 40 & 3 & 77.5 & & 12.5 & 10.0 & & & & & 0.25 & 0.09 & 0.383 & 0.0158 & $0.1041 \mathrm{NS}$ \\
\hline Waghauser & Germany & 17 & 34 & 4 & 88.2 & & 5.9 & & 5.9 & & & & 0.12 & 0.06 & 0.221 & 0.0065 & $-1.2122 \mathrm{NS}$ \\
\hline Nussloch & Germany & 20 & 40 & 6 & 35.0 & 20.0 & 2.5 & 10.0 & & & 22.5 & 10.0 & 0.80 & 0.71 & 0.786 & 0.0314 & $0.2216 \mathrm{NS}$ \\
\hline Plombieres & Belgium & 19 & 38 & 7 & 55.3 & 18.4 & 2.6 & 15.8 & 2.6 & 2.6 & & 2.6 & 0.53 & 0.17 & 0.650 & 0.0182 & $-0.9362 \mathrm{NS}$ \\
\hline Schinveld & The Netherlands & 20 & 40 & 6 & 60.0 & 2.5 & 5.0 & 7.5 & 12.5 & 12.5 & & & 0.60 & 0.41 & 0.615 & 0.0136 & -0.6444 NS \\
\hline Stolberg & Germany & 19 & 38 & 5 & 50.0 & 10.5 & 7.9 & 26.3 & & 5.3 & & & 0.74 & 0.57 & 0.678 & 0.0220 & $0.9764 \mathrm{NS}$ \\
\hline Warminster & Great-Britain & 20 & 40 & 4 & 85.0 & & 10.0 & 2.5 & 2.5 & & & & 0.20 & 0.35 & 0.273 & 0.0102 & $-1.1061 \mathrm{NS}$ \\
\hline Avonmouth & Great-Britain & 20 & 40 & 6 & 65.0 & 2.5 & 5.0 & 20.0 & & 2.5 & 2.5 & 2.5 & 0.60 & 0.15 & 0.546 & 0.0198 & -1.0798 NS \\
\hline Ringarum & Sweden & 20 & 40 & 4 & 77.5 & 2.5 & 12.5 & & & & & & 0.40 & 0.69 & 0.387 & 0.0147 & $-0.4261 \mathrm{NS}$ \\
\hline Gusum & Sweden & 20 & 40 & 5 & 67.5 & 5.0 & 7.5 & 15.0 & 5.0 & & & & 0.40 & 0.13 & 0.524 & 0.0169 & 0.0089 NS \\
\hline Norre Snede & Denmark & 20 & 40 & 4 & 85.0 & 2.5 & 2.5 & 10.0 & & & & & 0.20 & 0.35 & 0.273 & 0.0095 & $-1.4626 \mathrm{NS}$ \\
\hline \multirow[t]{2}{*}{ Hygum } & Denmark & 20 & 40 & 4 & 72.5 & & 17.5 & 7.5 & 2.5 & & & & 0.55 & 0.69 & 0.449 & 0.0175 & 0.4793 NS \\
\hline & & 290 & 580 & 8 & & & & & & & & & & & 0.522 & 0.01709 & \\
\hline
\end{tabular}

Abbreviations: Ho, observed heterozygosity; HW, Hardy-Weinberg; NS: not significant.

Polluted sites had specific combinations of increased metal concentrations: Stolberg, Plombieres and Noyelles (Cu, Pb, Zn, Cd), Avonmouth, Nussloch $(\mathrm{Pb}, \mathrm{Zn}, \mathrm{Cd})$, Hoboken $(\mathrm{Pb}, \mathrm{Cd})$, Gusum $(\mathrm{Cu}, \mathrm{Zn})$, Hygum $(\mathrm{Cu})$.

Metal stressed populations are given in bold.

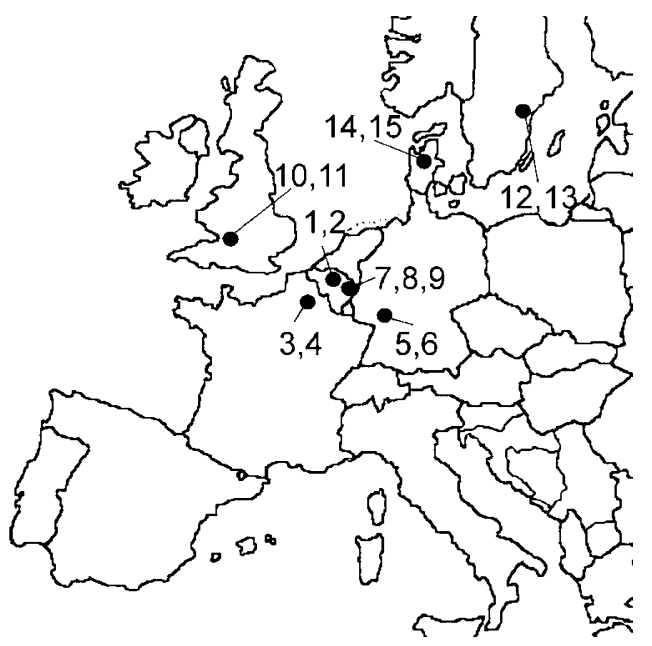

Figure 1 Sample locations of 15 European O. cincta populations. (1) Brasschaat, (2) Hoboken, (3) Hecq, (4) Noyelles, (5) Wag-Hauser Wald, (6) Nussloch, (7) Plombieres, (8) Schinveld, (9) Stolberg, (10) Warminster, (11) Avonmouth, (12) Ringarum, (13) Gusum, (14) Norre Snede and (15) Hygum.

matches completely only with the A and B alleles. A $5^{\prime}$ extension of the $m t$ promoter region revealed single nucleotide polymorphisms in the primer site. The bold underlined $\mathrm{G}$ in the MTF primer is a C in alleles C, D, E, $\mathrm{F}$ (no information available for alleles $\mathrm{G}$ and $\mathrm{H}$ ), the bold underlined $\mathrm{A}$ is a $\mathrm{C}$ in allele $\mathrm{F}$ and the bold underlined $\mathrm{C}$ is an A in some D alleles (Janssens et al., unpublished data). This may have resulted in a PCR bias in favor of alleles $\mathrm{A}$ and $\mathrm{B}$, both for the metal-stressed and reference populations.

\section{SSCP, RFLP and sequencing}

Allele frequencies of the different populations were resolved with single-stranded conformation polymor- phism (SSCP). For SSCP analysis, $7 \mu$ l PCR product was loaded on a non-denaturing polyacrylamide gel $(8 \%$ polyacrylamide gels containing 5\% glycerol). Products obtained using primer pairs MTF-184R and 163F-265R were loaded on one gel and each gel consisted of one population only. SSCP gels were run on a DCode universal mutation detection system (Biorad, Hercules, CA, USA) $(16 \mathrm{~h} / 150 \mathrm{~V})$ in a climate room $\left(5^{\circ} \mathrm{C}\right)$, keeping the temperature of the gel between 8 and $10^{\circ} \mathrm{C}$. Bands were visualized using SYBR gold staining dye (Invitrogen, Molecular Probes, Eugene, OR, USA). In some cases, it proved to be complicated to distinguish between some of the alleles. Four alleles were not resolved using SSCP, and were subjected to restriction fragment length polymorphism (RFLP) analysis with MseI (see below). Restricted products were visualized on an ALF express automated sequences (Amersham Pharmacia, Piscataway, NJ, USA) using Cy5.0-labelled primers. Of each SSCP gel, PCR products that revealed different banding patterns (for at least one of the primer pairs used) were directly sequenced (homozygotic individuals) or cloned in pGemT vectors (Promega) (heterozygotic individuals) and sequenced by a sequencing service (ServiceXS, Leiden, The Netherlands). Multiple representatives of each of the observed alleles were sequenced. However, cloning reveals the errors made by DNA polymerases (Paabo and Wilson, 1988). The error rate of Taq polymerase is about 1:1000 bases (Villablanca et al., 1998). Therefore, clones from one PCR reaction may differ. To discriminate real alleles from Taq errors several clones from each PCR reaction have to be sequenced. For heterozygote animals, this requires six sequencing reactions; three for the first and three for the second allele (Villablanca et al., 1998). We therefore chose to follow a different approach: we identified and resequenced several singletons. In total, 17 singletons were observed, from eight singletons new clones were sequenced (on an ALF express automated sequencer, 
Amersham) to determine whether it really were different alleles or whether differences were misincorporation errors. All eight turned out to be Taq errors, which led us to consider all other singletons as misincorporations as well. However, it has to be mentioned that this is a simplification that may underestimate the actual number of alleles, resulting in a more conservative estimate of allelic diversity. It appeared that all the alleles that were resolved using SSCP could easily be distinguished by a combination of differences in fragment length and RFLP, therefore four populations were not subjected to SSCP (Gusum, Ringarum, Hygum, Norre Snede). Those populations were analyzed by RFLP only. First PCR fragment lengths were determined on an ALF express automated sequencer. Afterwards, alleles of equal size were digested using MseI, as described by the manufacturer (New England Biolabs, Ipswich, MA, USA). The size of the digested PCR products was again resolved on an ALF express automated sequencer using labeled forward or labeled reverse primers. Sequences are deposited in GenBank under the Accession nos. AY182010 and DQ645813-DQ645819.

\section{Data analysis}

Sequences of the $m t$ gene were aligned using Genedoc (Nicholas and Nicholas, 1997). To visualize relationships among alleles, a statistical parsimony network was constructed using a maximum parsimony criterion, as implemented in TCS version 1.13 (Clement et al., 2000). All insertions/deletions were ignored when the statistical parsimony network was constructed. The resulting network gives a clear representation of the number of mutations distinguishing the different alleles. We visualized the distribution of alleles among metal-stressed and non-stressed populations in this network. To test for Hardy-Weinberg equilibrium expectations, the allelic composition of each population was evaluated using the exact test as implemented in Genepop 3.2 (Raymond and Rousset, 1995). To visualize the relationships among populations without forcing them in a bifurcating tree, we conducted a principal component analysis (PCA) using the software package PCAGEN (available at http:/ / www.unil.ch/izea/softwares/pcagen.html; J Goudet). To detect the influence of selection on the $m t$ gene several additional tests were applied. At the gene level, aminoacid substitution mutations versus silent mutations were analyzed. For all pairs of alleles, we calculated the ratio of $d_{n}$ (the number of substitutions causing amino-acid replacements divided by the total number of possible non-synonymous sites) to $d_{s}$ (the number of silent substitutions divided by the number of possible synonymous sites), using the modified Nei and Gojobori (1986) method with pairwise deletion of gaps. Calculations were carried out in the program MEGA version 2.1 (Kumar et al., 2004). Deviations of $d_{n} / d_{s}$ from unity indicate selective forces are acting on the gene, with $d_{n} / d_{s}<1$ indicating purifying selection, and $d_{n} / d_{s}>1$ indicating positive selection. The null hypothesis $d_{n}=d_{s}$ was tested using a one-tailed Fisher's exact test for positive selection, as implemented in MEGA version 2.1 (Kumar et al., 1993). At the population level, Tajima's test (Tajima, 1989) was used to determine whether the $m t$ gene of $O$. cincta is evolving neutrally. Significant departures of Tajima's $D$ from zero were evaluated using the program DNASP
(Rozas et al., 2003). Furthermore, to explore whether allele frequencies were different between metal-stressed and reference populations, a genetic structure analysis (AMOVA) was applied, as implemented in the program ARLEQUIN (Schneider et al., 2000). AMOVA was used to infer proportions of variation among metal-stressed and non-stressed populations. To test whether this grouping explains more variance than any other random clustering, the populations were randomly divided in two groups of seven and eight populations and AMOVA was applied. This procedure was repeated 5040 times to obtain the distribution of variance among groups under the null hypothesis of no effect of soil pollution.

Furthermore, a goodness of fit test (G-test) was performed on allele frequencies to test whether alleles are homogenously distributed over metal-stressed and reference populations. In addition, to gain more insight into which allele might be affected by environmental metal stress, the allele frequencies were correlated with metal pollution. As previous studies showed that expression of $m t$ mRNA of $O$. cincta is induced after exposure to cadmium (e.g. Sterenborg and Roelofs, 2003) and lead (Timmermans, unpublished data), allele frequencies were plotted against soil cadmium and lead concentrations of the different locations. The soil concentrations of those metals were combined into one 'pollution index' as described by Widianarko et al. (2000). Metal concentrations from each site were divided by the Dutch maximum permissible concentrations (MPC). The values obtained for the separate metals were multiplied and the logarithm of the product was taken. Spearman's rank correlation was used to test for correlation between this pollution index and allele frequencies (only alleles occurring with a frequency of $5 \%$ or more in at least four of the eleven populations were included in the analysis to prevent biases owing to small sample size). It has to be mentioned that the frequencies of the different alleles are not completely independent from each other (if the frequency of one allele is going up, one or more others have to go down); therefore the results of the Spearman's rank correlations should be interpreted with care.

Finally, as directional selection is often assumed to deplete genetic diversity (owing to the replacement of other segregating alleles by the selected allele; Schlotterer, 2003), genetic diversity within the 15 populations was assessed. Gene diversity (=haplotype diversity) and nucleotide diversity (Nei, 1987) were estimated. We evaluated whether measures of diversity were correlated with soil pollution using Spearman's rank correlation. An improved Bonferroni procedure was applied to correct for multiple testing (Haccou and Meelis, 1992).

\section{Results}

\section{Variation in the $m t$ gene}

A total of 290 Northwest (NW)-European specimens originating from 15 populations were analyzed. The amplified fragment had a length of $364 \mathrm{bp}$, including primers. The $m t$ gene of $O$. cincta appeared highly variable. Within NW-Europe eight alleles with a total of 51 polymorphic sites were observed. The coding region had 13 variable sites. One insertion/deletion (indel) was observed in the coding region, with a length of six nucleotides. A total of 26 polymorphic sites were found 


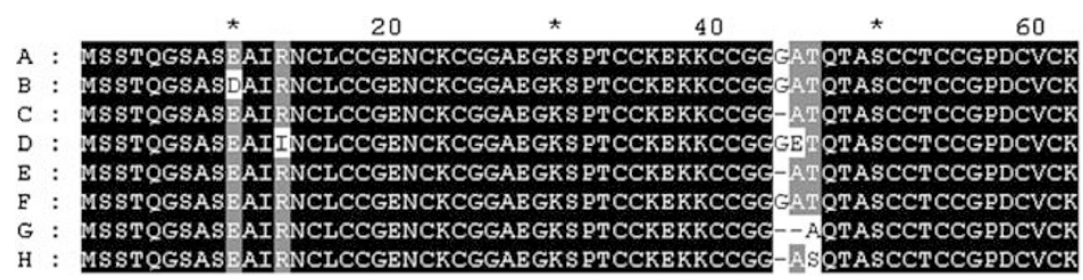

Figure 2 Aligned $m t$ amino-acid sequences inferred from the different alleles. The sequences represent amino acids 1-62 from the 77 amino acids of the complete protein.

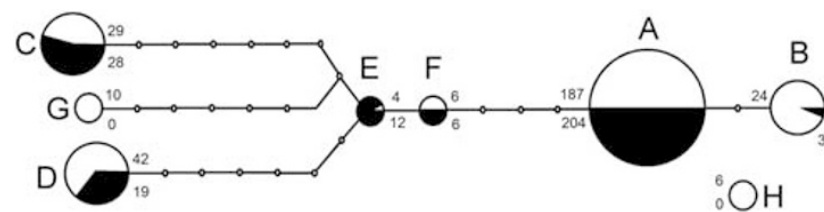

Figure 3 Haplotype-tree of the $m t$ gene of $O$. cincta (excluding gaps) constructed using TCS. Eight haplotypes were observed $(\mathrm{A}-\mathrm{H})$. Each haplotype shows the proportion to which the allele is observed in a metal-stressed (white) and a reference population (black). Numbers indicate how many times an allele was observed: for metal-stressed populations above and for reference populations below. Allele $\mathrm{H}$ could not parsimoniously be connected to the network.

in the intron of the gene (one indel of eight nucleotides). The $5^{\prime}$ untranscribed region had 12 variable sites, with three single nucleotide insertions/deletions. Translation of sequences to the amino-acid level revealed five amino acids of 62 to be variable (amino-acid positions 10, 13, 44, 45,46 of allele A; Figure 2). The haplotype tree (Figure 3) shows that allele $\mathrm{A}$ is overall predominantly present, that alleles B and D are most often observed in metal-stressed populations, and that allele E mainly occurs in reference locations. The predominant presence of allele $\mathrm{A}$ is in accordance with the data of Roelofs et al. (2006).

Genetic diversity and genetic structure in relation to metal contamination

First, a PCA was conducted to visualize relationships among populations. PCA did not cluster stressed populations separately from reference populations (Figure 4). Second, we estimated the rates of synonymous and non-synonymous changes between all pairs of alleles, and tested the differences using Fisher's exact test. Several comparisons revealed $d_{n} / d_{s}$ ratios larger than one (most comparisons with allele D); however, the null hypothesis of neutral evolution could not be rejected in any of the pairwise comparisons made (data not shown). Third, at the population level, Tajima's test was used to determine whether $m t$ is evolving neutrally. The null hypothesis of neutral evolution could not be rejected for any of the populations tested (Table 1) implying, according to these tests, that the gene is evolving in a neutral manner. Fourth, we analyzed genetic differentiation among metal-stressed and non-stressed populations and determined whether metal-related shifts in allele frequencies occurred. AMOVA assigned a small but significant amount of the variance to variation among metal-stressed and non-stressed populations (2.11\%; $P=0.04$; Table 2). Furthermore, a goodness-of-fit test (G-test) rejected the null hypothesis that alleles are homogenously distributed between metal-stressed and

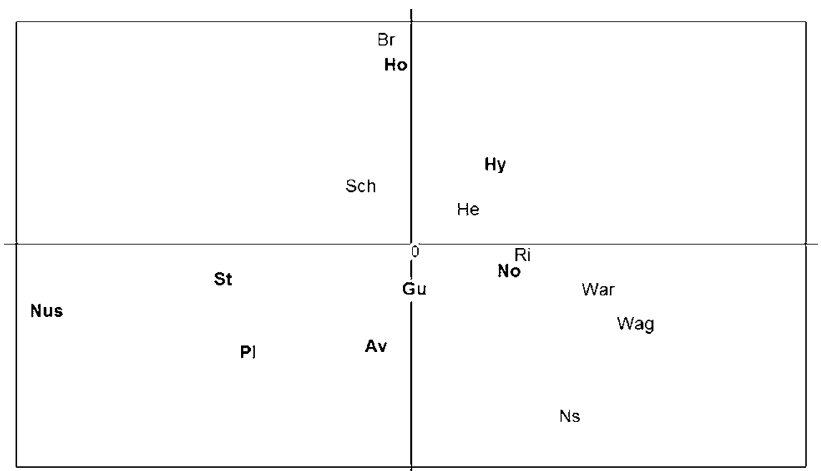

Figure 4 PCA showing the genetic relationship among populations. PCA axis 1 explains $66.6 \%$ of the variance, PCA axis 2 explains $12.4 \%$ and PCA axis 3 (not shown) $10.6 \%$ the variance. Metalstressed populations are shown in bold.

reference populations $(P<0.01)$. To gain insight into which alleles might be responsible for this outcome, the allele frequencies were correlated with metal pollution. Only alleles that were present at $5 \%$ or more in at least four of the populations were analyzed (alleles A to D). Spearman's rank correlation revealed a negative correlation between the frequency of allele $\mathrm{A}$ and a positive correlation between the frequency of allele $\mathrm{D}$ and the combined cadmium and lead concentrations in the soil. However, this latter result was not significant after improved Bonferroni correction (Figure 5). Finally, genetic diversity within the metal-stressed and reference populations was assessed. Overall haplotype diversity and overall nucleotide diversity of NW-European samples were 0.522 and 0.0171 , respectively. As diversity varied greatly between populations (Table 1), we also evaluated whether measures of diversity were correlated with soil cadmium and lead concentrations. Spearman's rank correlation revealed positive correlations between soil metal concentrations and nucleotide and haplotype diversity (Figure 5).

\section{Discussion}

The number of studies reporting evidence for (positive directional) selection in gene sequences has grown remarkably, primarily owing to the advances in DNA sequencing (Ford, 2002) and the advances in statistical methods for detecting adaptation (Yang and Bielawski, 2000). Although studies applying statistical tests can help us to estimate the likelihood of selection at a certain locus, they do not necessarily add to our understanding of the mechanisms through which natural selection 
Table 2 AMOVA for partitioning variation among populations, based on Fst estimated from $m t$ allele frequencies

\begin{tabular}{|c|c|c|c|c|c|}
\hline Source of variation & $d f$ & Sum of squares & Variance components & Percentage of variation & P-value \\
\hline Among groups & 1 & 2.296 & 0.00559 & 2.11 & 0.036 \\
\hline Among populations within groups & 13 & 8.836 & 0.01118 & 4.23 & $<0.001$ \\
\hline Within populations & 565 & 139.975 & 0.24774 & 93.66 & $<0.001$ \\
\hline Total & 579 & 151.107 & 0.26451 & & \\
\hline
\end{tabular}

Abbreviations: AMOVA, analysis of molecular variance; $m t$, metallothionein.

Populations were grouped in metal-stressed and reference populations, significance test was based on 5040 permutations.
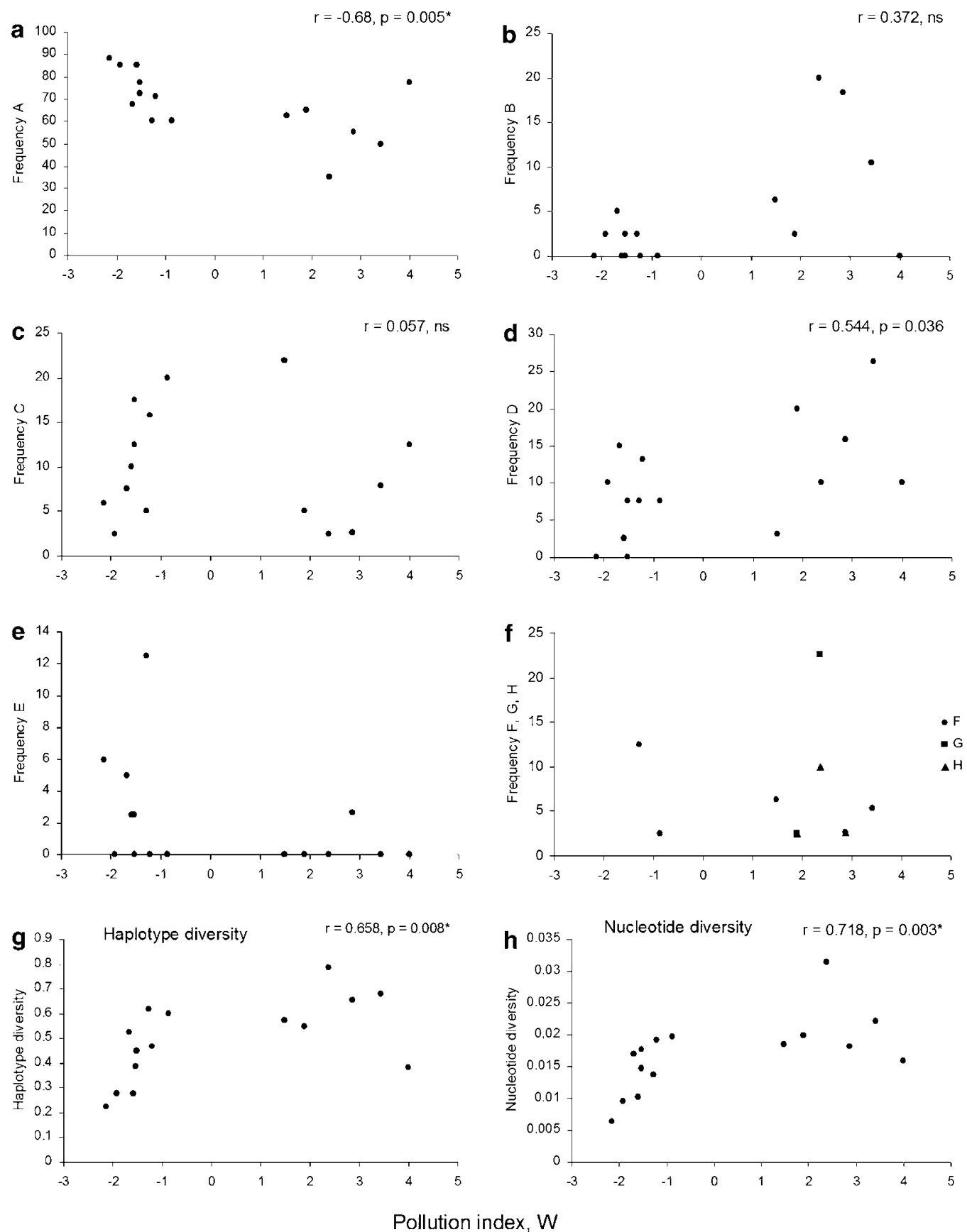

Figure $5(\mathbf{a}-\mathbf{f})$ Allele frequencies (\%) and ( $\mathbf{g}$ and $\mathbf{h}$ ) haplotype and nucleotide diversity of the different populations, plotted against pollution index, W (a combined measure for soil cadmium and lead concentrations). Spearman's rank correlation was used to test for correlation. *Indicates significance at the 0.05 level after improved Bonferroni correction. NS: not significant. 
promotes adaptation. Linking adaptive processes to selection regimes in the wild is only feasible when such statistical tests are applied in combination with field observations. We analyzed a gene of known function, combining field data with statistical tests on sequence differences. We assessed whether the $m t$ gene of $O$. cincta is subject to selection by investigating alleles and allele frequencies among metal-stressed and reference populations.

In total, eight highly divergent alleles were resolved in NW Europe. At the nucleotide level, a total of 51 polymorphic sites (including five amino-acid changes) were observed. Several statistical tests commonly used to unravel positive selection were applied. A Fisher's exact test, examining whether $d_{n}$ is significantly greater than $d_{s}$, revealed no positive selection among alleles. This, of course, does not necessarily mean that there have been no amino-acid substitutions driven by positive selection. The rate of $d_{n} / d_{s}$ does not tell us whether nonsynonymous substitutions actually resulted in functional changes of the gene. However, none of the five aminoacid replacements that we observed is expected to cause large differences in metal-binding capacities of the protein: three of the amino-acid replacements (positions 44, 45, 46 of Hensbergen et al., 1999) are observed outside the two metal-binding clusters. Furthermore, the positions of all the cysteines, which in the tertiary structure form the metal-thiolate clusters (Kagi and Schaffer, 1988), are conserved.

At the population level, Tajima's test revealed that the null hypothesis of neutral evolution could not be rejected for any of the populations analyzed. However, it has to be mentioned that Tajima's test is not very strong (Nei and Kumar, 2000) and that not all its assumptions may have been fulfilled. One of the assumptions of Tajima's test is that all nucleotides mutate equally. It is obvious that this is not the case for coding DNA regions (Nei and Kumar, 2000). Based on those statistical tests, one has to conclude that the sequence differences revealed are not affected by selective forces from environmental pollution.

However, allele frequencies in the field weakly suggest the presence of traces of natural selection. Although a PCA did not show stressed populations clustering separately from reference populations, a G-test rejected the null hypothesis that alleles are homogeneously divided over metal-stressed and reference populations. Furthermore, an AMOVA assigned, in addition to a large amount of variation among populations within groups, a significant amount of the variance to variation between metal-stressed and non-stressed populations. The grouping into metal-stressed and reference populations explained more variance than random clusterings of the populations into two groups of seven and eight. The suggestion that this partitioning is due to environmental metal pollution is strengthened by a recent microsatellite study on those 15 springtail populations. An AMOVA analysis based on six microsatellite markers did not assign any significant amount of the variance to variation between the metal-stressed and the reference populations (Timmermans et al., unpublished data). This implies that, in contrast with the $m t$ data presented here, (neutral) microsatellite variation is not affected by environmental metal pollution. Finally, alleles B and D were predominantly observed in metal-stressed popula- tions and allele A was negatively correlated with soil pollution. This suggests that in metal-polluted environments allele $\mathrm{A}$ is replaced by other segregating alleles.

Although we cannot rule out that genetic drift and/or historical factors have generated the observed pattern, the results suggest that selection is affecting the $m t$ locus. As we do not see significant changes in the primary structure of the protein, apparently selection does not act on the coding region of the gene itself, but on a region surrounding it. Linkage of the coding region to adjacent advantageous polymorphisms may have resulted in increased frequencies of alleles B and D and to increased differentiation at this locus (e.g. Slatkin and Wiehe, 1998; Schlenke and Begun, 2004). Recent studies on metal tolerance in $O$. cincta have revealed differences in $m t$ mRNA expression between populations from metalstressed and reference locations (e.g. Timmermans et al., 2005b). The metal-stressed Plombières population appeared to have higher $m t$ mRNA expression upon exposure to cadmium than a reference population (Sterenborg and Roelofs, 2003). These differences are presumably caused by polymorphisms in the promoter region of the $m t$ gene (Janssens, unpublished data). Roelofs et al. (2006) showed increased occurrence of promoter alleles B and D1/D2 (which are linked to allele $\mathrm{B}$ and D in our study respectively) in families exhibiting increased $m t$ expression. Tight linkage of polymorphisms in the coding region to functional polymorphisms in the promoter region of the $m t$ gene, most likely resulted in the patterns observed.

The question arises why the population differentiation at this locus between metal-stressed and reference populations is not more pronounced if $m t$ has a function in metal tolerance? Probably migration from neighboring (non-contaminated) locations has prevented a further increase of alleles $\mathrm{B}$ and $\mathrm{D}$, resulting in a 'migration load'. Most contaminated sites are small islands in a more or less 'pristine' environment. It is often shown and suggested that gene flow from adjacent populations obscures genetic effects of local stressors (e.g. Ingvarsson, 2001; Theodorakis, 2001; Colson, 2002; Lenormand, 2002). Therefore, only strong selection regimes will result in very clear associations between allele frequencies and environmental stress (Vasemagi and Primmer, 2005). This implies that, even if selection does act on the $m t$ gene of $O$. cincta and selective effects are present that they may remain hard to detect owing to the homogenizing effects of gene flow between reference and metal-stressed populations (migration-selection balance). Although, $O$. cincta is a wingless, soil-dwelling species that is highly diversified over Europe (Timmermans et al., 2005a), several studies have inferred high gene flow between O. cincta populations (Frati et al., 1992; Van der Wurff et al., 2003). Possibly this high rate of exchange of genetic material between neighboring populations maintains diversity and counteracts shifts in allele frequencies between metal-stressed and reference populations. Such gene flow could explain the positive correlation between pollution and diversity (Figure 4). A comparable hypothesis was also suggested by Lange et al. (1990) for D. melanogaster. Lange et al. (1990) attempted to relate $M t n$ duplication (which was shown to increase metal tolerance in D. melanogaster), to environmental metal contamination. However, they 'failed to detect any consistent association of the frequencies of the 
Mtn duplications with heavy metal exposure', and suggested that migration of animals from non-contaminated regions swamped differentiation in duplication frequencies (Lange et al., 1990).

The intriguing positive correlation between the soil metal concentrations and genetic diversity, which seems to be due to the reduced abundance of allele $A$, might have a different basis. The increased genetic diversity at the $m t$ locus in polluted sites might, for instance, be a result of overdominance or balancing selection: heterozygotes at the mt locus being superior to homozygotes in metal-polluted environments. Alternatively, metalpolluted environments may be more heterogenous than non-polluted ones. As metals are often patchily distributed and soil concentrations differ greatly from place to place, the metal-stress experienced by individuals at a polluted location is variable, resulting in different selection regimes. If a certain allele is advantageous at a highly polluted site, it might be less advantageous in a patch that is less polluted only $1 \mathrm{~m}$ away.

It seems that a functional study on the $m t$ of $O$. cincta, linking metal tolerance to mRNA expression levels and to DNA sequence and frequency data (information about promoter region and coding region) is necessary to test those hypotheses. Such a study will provide more insight into the role of the $m t$ gene in metal tolerance of $O$. cincta. The study presented here shows that field observations remain an important tool for understanding selection and adaptive processes in the wild.

\section{Acknowledgements}

We wish to thank Thierry Janssens, Dick Roelofs and two anonymous reviewers for their valuable comments on earlier versions of the manuscript.

\section{References}

Clement M, Posada D, Crandall KA (2000). TCS: a computer program to estimate gene genealogies. Mol Ecol 9: 1657-1659.

Colson I (2002). Selection and gene flow between microenvironments: the case of Drosophila at Lower Nahal Oren, Mount Carmel, Israel. Mol Ecol 11: 1311-1316.

Daborn PJ, Yen JL, Bogwitz MR, Le Goff G, Feil E, Jeffers S et al. (2002). A single P450 allele associated with insecticide resistance in Drosophila. Science 297: 2253-2256.

Ford MJ (2002). Applications of selective neutrality tests to molecular ecology. Mol Ecol 11: 1245-1262.

Frati F, Fanciulli PP, Posthuma L (1992). Allozyme variation in reference and metal-exposed natural populations of Orchesella cincta (Insecta:Collembola). Biochim Systemat Ecol 20: 297-310.

Haccou P, Meelis E (1992). Statistical Analysis of Behavioural Data. Oxford University Press: Oxford.

Hensbergen PJ, Donker MH, van Velzen MJM, Roelofs D, van der Schors RC, Hunziker PE et al. (1999). Primary structure of a cadmium-induced metallothionein from the Insect Orchesella cincta (Collembola). Eur J Biochem 259: 197-203.

Ingvarsson PK (2001). Restoration of genetic variation lost - the genetic rescue hypothesis. Trends Ecol Evol 16: 62-63.

Kagi JHR, Schaffer A (1988). Biochemistry of metallothionein. Biochemistry 27: 8509-8515.

Kumar S, Tamura K, Nei M (2004). MEGA3: integrated software for molecular evolutionary genetics analysis and sequence Alignment. Brief Bioinform 5: 150-163.

Lange BW, Langley CH, Stephan W (1990). Molecular evolution of the Drosophila metallothionein genes. Genetics 126: 921-932.
Lenormand $\mathrm{T}$ (2002). Gene flow and the limits to natural selection. Trends Ecol Evol 17: 183-189.

Lenormand T, Bourguet D, Guillemaud T, Raymond M (1999). Tracking the evolution of insecticide resistance in the mosquito Culex pipiens. Nature 400: 861-864.

Liu N, Scott JG (1998). Increased transcription of CYP6D1 causes cytochrome P450 mediated insecticide resistance in house fly. Insect Biochem Mol Biol 28: 531-535.

Maroni G, Wise J, Young JE, Otto E (1987). Metallothionein gene duplications and metal tolerance in natural populations of Drosophila melanogaster. Genetics 117: 739-744.

Nachman MW, Hoekstra HE, D'Agostino SL (2003). The genetic basis of adaptive melanism in pocket mice. Proc Natl Acad Sci USA 100: 5268-5273.

Nei M (1987). Molecular Evolutionary Genetics. Columbia University Press: New York.

Nei M, Gojobori T (1986). Simple methods for estimating the numbers of synonymous and nonsynonymous nucleotide substitutions. Mol Biol Evol 3: 418-426.

Nei M, Kumar S (2000). Molecular Evolution and Phylogenetics. Oxford University Press: New York.

Nicholas KB, Nicholas HB (1997). Genedoc: a tool for editing and annotating multiple sequence alignments. Distributed by the author.

Paabo S, Wilson AC (1988). Polymerase chain-reaction reveals cloning artifacts. Nature 334: 387-388.

Posthuma L, van Straalen NM (1993). Heavy metal adaptation in terrestrial invertebrates: a review of occurrence, genetics, physiology and ecological consequences. Comp Biochem Physiol 106c: 11-38.

Raymond M, Berticat C, Weill M, Pasteur N, Chevillon C (2001). Insecticide resistance in the mosquito Culex pipiens: what have we learned about adaptation? Genetica 112: 287-296.

Raymond M, Rousset F (1995). GENEPOP (version 1.2) population genetics software for exact tests and ecumenicism. J Hered 86: 248-249.

Roelofs D, Overhein L, de Boer ME, Janssens TKS, van Straalen NM (2006). Additive genetic variation of transcriptional regulation: metallothionein expression in the soil insect Orchesella cincta. Heredity 96: 85-92.

Rozas J, Sanchez-DelBarrio JC, Messequer X, Rozas R (2003). DnaSP, DNA polymorphism analyses by the coalescent and other methods. Bioinformatics 19: 2496-2497.

Schlenke TA, Begun DJ (2004). Strong selective sweep associated with a transposon insertion in Drosophila simulans. Proc Natl Acad Sci USA 101: 1626-1631.

Schlotterer C (2003). Hitchhiking mapping - functional genomics from the population genetics perspective. Trends Genet 19: $32-38$.

Schneider S, Roessli D, Excoffier L (2000). Arlequin: a Software for Population Genetics Data Analysis. Ver 2.000. Genetics and Biometry Lab, Department of Anthropology, University of Geneva.

Slatkin M, Wiehe T (1998). Genetic hitch-hiking in a subdivided population. Genet Res 71: 155-160.

Sterenborg I, Roelofs D (2003). Field-selected cadmium tolerance in the springtail Orchesella cincta is correlated with increased metallothionein mRNA expression. Insect Biochem Mol Biol 33: 741-747.

Tajima F (1989). Statistical method for testing the neutral mutation hypothesis by DNA polymorphism. Genetics 123: 585-595.

Theodorakis CW (2001). Integration of genotoxic and population genetic endpoints in biomonitoring and risk assessment. Ecotoxicology 10: 245-256.

Timmermans M, Ellers J, Marien J, Verhoef SC, Ferwerda EB, Van Straalen NM (2005a). Genetic structure in Orchesella cincta (Collembola): strong subdivision of European populations inferred from mtDNA and AFLP markers. Mol Ecol 14: 2017-2024. 
Timmermans M, Ellers J, Roelofs D, Van Straalen NM (2005b). Metallothionein mRNA expression and cadmium tolerance in metal-stressed and reference populations of the springtail Orchesella cincta. Ecotoxicology 14: 727-739.

Van der Wurff AWG, Isaaks JA, Ernsting G, Van Straalen NM (2003). Population substructures in the soil invertebrate Orchesella cincta, as revealed by microsatellite and TE-AFLP markers. Mol Ecol 12: 1349-1359.

Vasemagi A, Primmer CR (2005). Challenges for identifying functionally important genetic variation: the promise of combining complementary research strategies. Mol Ecol 14: 3623-3642.
Villablanca FX, Roderick GK, Palumbi SR (1998). Invasion genetics of the Mediterranean fruit fly: variation in multiple nuclear introns. Mol Ecol 7: 547-560.

Widianarko B, Verweij RA, van Gestel CAM, van Straalen NM (2000). Spatial distribution of trace metals in sediments from urban streams of Semarang, Central Java, Indonesia. Ecotoxicol Environ Saf 46: 95-100.

Wray GA, Hahn MW, Abouheif E, Balhoff JP, Pizer M, Rockman MV et al. (2003). The evolution of transcriptional regulation in eukaryotes. Mol Biol Evol 20: 1377-1419.

Yang ZH, Bielawski JP (2000). Statistical methods for detecting molecular adaptation. Trends Ecol Evol 15: 496-503. 